\title{
Fusion algorithm based on W-transfrom and an improved local energy
}

\author{
XU Nuo ${ }^{1, a}$,WANG Lu ${ }^{1, b}$, ZHAO Ke ${ }^{2}$,PI Sai-nan ${ }^{1}$,WANG Xiao-chun ${ }^{1, c^{*}}$ \\ ${ }^{1}$ College of Science, Beijing Forestry University, Beijing100083, China \\ ${ }^{2}$ College of Forestry, Beijing Forestry University, Beijing100083, China \\ a506599632@qq.com , ${ }^{b}$ luer92@163.com , cwamgxiao@bjfu.edu.cn
}

\begin{abstract}
Keywords: W-system Image Fusion Fusion rules Objective evaluation index
Abstract. W-transform is a new kind of orthogonal transform which provides a method to eliminate the phenomenon of Gibbs. This paper proposes a new fusion algorithm based on the W-transform. Compared to the classical fusion algorithm based on DCT and Haar transform, the algorithm based on the $\mathrm{W}$-transform proposed in this article is improved in four evaluation index including the standard deviation, information entropy, average gradient, and spatial frequency. This paper also presents an improved fusion rule according to the characteristics of low frequency and high frequency components obtained by the $\mathrm{W}$-transform, it adopts weighted average method in the low frequency part by introducing information entropy as the weights, while combines local region features and each single pixel value in the high frequency band. Compared to the fusion rule based only on local region energy, the improved fusion rule can effectively improve the objective evaluation index and visual effect of the fused image. Experimental results reveal the superiority of algorithm proposed in this paper compared to comparable algorithms.
\end{abstract}

\section{Introduction}

The concept of image information fusion is first appeared in the late1980s [1]. In computer vision, image fusion is the process of producing a single image from two or more images which are obtained by multiple image sensors with same scene and different resolutions. The fused image should have more accurate, more objective, more consistent image description which is more useful for human or machine perception. In recent years, image fusion has become one of the hot topics in the field of image processing, and fusion technology is widely used in field of medical image processing [2], traffic guidance [3], military, remote sensing [4], machine vision ,biology [5] and so on.

Image fusion can be performed at three levels: pixel level, feature level and decision level. The most frequently used methods for image fusion are based on pixel level. Pixel level fusion methods can be broadly divided into two groups: spatial domain fusion and transform domain fusion. The spatial domain methods, such as averaging and principal component analysis (PCA), deal with the image pixels directly without any preprocessing or post processing, they are easy to produce spatial distortion. The transform domain techniques are based on coefficients of transformed image, and fusion is then applied by combining their transform coefficients under various fusion rules. Transform domain methods including the Pyramid decomposition method [6], the wavelet transform [7], and the discrete cosine transform [8] show a better performance in spatial and spectral quality of the fused image compared to other spatial fusion methods, but they are prone to produce blockiness in image edges and directional information can be captured is limited. To overcome these drawbacks, curvelet [9] framelet, and contourlet [10] based techniques are employed, but these approaches are computationally expensive and also bring error and artifacts due to the pseudo-Gibbs phenomenon. However, for human observer or following processing stages, the fusion scheme must not introduce any distortion or inconsistencies. In this paper, the fusion algorithm based on the W-system, a new hybrid function system, is proposed, it calculates the coefficients with respect to the W-transform to each source image at first and then calculate the 
transform coefficients of the fused image based on some fusion rule, which is computationally simple and can also efficiently eliminate the artifacts due to the Gibbs phenomenon.

Wavelet analysis is a new subject in the field of mathematics, and the Haar wavelet [11], a sequence of rescaled "square-shaped" functions, is one of the earliest used compactly supported orthogonal wavelet. The Haar wavelet function has many features, such as simple structure, convenient calculation and linear phase of the corresponding filter, it has become a useful mathematical tool for a variety of signal and image processing applications. But because of its discontinuity in the time domain, it may appear step shape when it is used to process the discontinuous information. DCT for Discrete Cosine Transform is also a kind of orthogonal transformation. DCT has a powerful ability on information concentration, which plays an important role in image processing, but DCT is prone to blocking artifact, which can lead to a loss of image quality.

Based on a new class of orthogonal function system, the W-system [12], this article proposes a new fusion algorithm. W-transform is an orthogonal transformation denoted discretely by basis functions of $\mathrm{W}$-system. As the $\mathrm{W}$-system has basis functions with different levels of discontinuity, the W-transform has the advantage on the discontinuous part of gray value, it can effectively overcome pseudo-Gibbs phenomenon [13] generated by harmonic transformations such as Fourier or DCT transform. This paper also presents an improved fusion rules according to the characteristic of high frequency and low frequency. In the high frequency part, the absolute value of the coefficients and the energy of coefficient window are combined so that the greatest pixel value or energy is assigned to the corresponding pixel, which is improvement of the fusion rule only based on local area energy [14]. In the low frequency part, we introduce the information entropy of the corresponding spatial domain as weight, and use the weighted average method instead of the original algorithm to maintain the rich information of the original image, because information entropy can be used to measure how much information there is in an image. The improvement of low frequency and high frequency makes effectively improvement on fusion effect of orthogonal transformation based on Haar, DCT and W- system. Experimental results reveal the superiority of algorithm proposed in this paper compared to comparable algorithms.

\section{W-system}

W-system is a new type of complete orthogonal functions on, composed of piecewise polynomial and constructed by Haar orthogonal function and Legendre polynomial. It is a generalization of Haar wavelet function system that the W-system of degree zero is the Haar function. The $k$ th W-system is defined as follows:

$$
W^{k}=\bigcup_{\mu=0}^{k} S_{N}^{\mu}, \quad N=2^{n}, \quad n=1,2,3,4, \mathrm{~L}
$$

In this formula, $S_{N}^{\mu}$ is a function set consisting of $\mathrm{N}$ functions, where function $s_{j}^{\mu}$ defined on [0,1] is a piecewise function whose formula in the interval $\left(\frac{j}{2^{n}}, \frac{j+1}{2^{n}}\right)$ is the multiplication of the $j$ th Haar function $h_{j}(x)$ and $P_{\mu}\left(2^{n} x-j\right)$ obtained by performing the dilation and translation to the $\mu$ th normalized Legendre polynomial $P_{\mu}(x)$, i.e.

$$
s_{j}^{\mu}(x)=P_{\mu}\left(2^{n} x-j\right) \times h_{j}(x), \quad x \in\left(\frac{j}{2^{n}}, \frac{j+1}{2^{n}}\right) \quad j=0,1, \mathrm{~L}, 2^{n}-1
$$

and 


$$
S_{N}^{\mu}=\left\{s_{0}^{\mu}, s_{1}^{\mu}, \mathrm{L}, s_{N-1}^{\mu}\right\}
$$

The first 32 basis functions of W-system is depicted in Fig. 1.
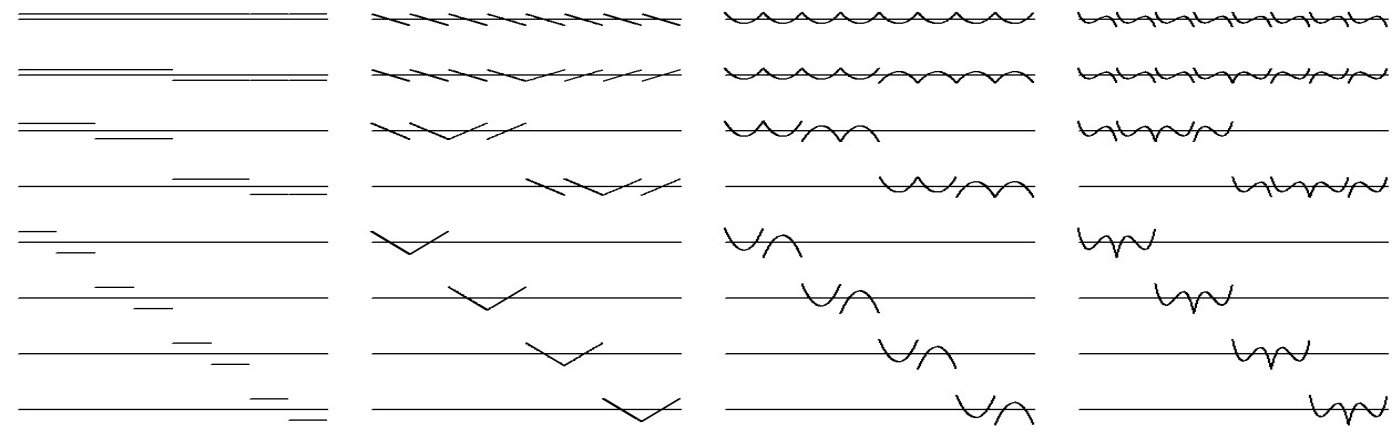

Figure 1 The first 32 basis functions of $\mathrm{W}$-system $(\mathrm{N}=8, \mathrm{k}=3)$

After discretizing the $\mathrm{W}$-system, the W-transformation matrix can be obtained. And the more specific introduction for $\mathrm{W}$-system and constructed methods of $\mathrm{W}$-transform matrix can be found in the reference [12].

Accordingly, the specific form of the $k$ th $\mathrm{W}$-transform is defined as

$$
P=W F W^{T}
$$

Where $F$ is the image matrix, $P$ is the corresponding spectrum of $F, W$ is the $k$ th $\mathrm{W}$-matrix with the same size as $F$. By the orthogonality of $W$, we can get $\mathrm{W}$-inverse transform:

$$
F=W^{T} P W
$$

\section{The image fusion algorithm based on W-system}

To construct the fusion algorithm based on the W-system, the source images are first decomposed by the W-transform, and then fusion takes place in the transform coefficients located at different scales.

The decomposition of W-system. (1) After registering and preprocessing the source images, all images have the size of $512 \times 512$. Divide the two source images A, B into several blocks with the same size, which is that dividing the pixel matrix of two images equally in row and column size to get a series of sub blocks with length " $d x$ " and width " $d y$ " separately, usually $d x=d y$. The $j$ th sub block of image A is denoted as $A_{j}(j=1,2, \mathrm{~L})$. (2) Decompose each sub block $I_{A}$ with W-transform to get its high frequency and low frequency. (3) The low-frequency components continue to be decomposed based on W-transform to get the decomposition spectrum of the second layer. Likewise, we can get a structure pattern decomposition with $n$ layers.

The $n$th layer of sub block $I_{A}$ of the source image $A$ is decomposed as follows, and the decomposition of original image $\mathrm{B}$ is similar.

$$
I_{A} \rightarrow\left\{{ }^{n} L L_{I_{A}},\left\{{ }^{i} L H_{I_{A}},{ }^{i} H H_{I_{A}},{ }^{i} H L_{I_{A}}\right\}_{i=1,2, L, n}\right\}
$$

Where ${ }^{i} \mathrm{LH}_{I_{A}},{ }^{i} \mathrm{HH}_{I_{A}},{ }^{i} \mathrm{HL}_{I_{A}}, i=1,2, \mathrm{~L}, n$ are horizontal, diagonal and vertical high frequency components of the $n$th layer decomposition of sub block $I_{A}$, that collectively referred to as high frequency components, and ${ }^{n} L L$ is low frequency component of the $n$th layer decomposition of sub block $A_{j}$. 


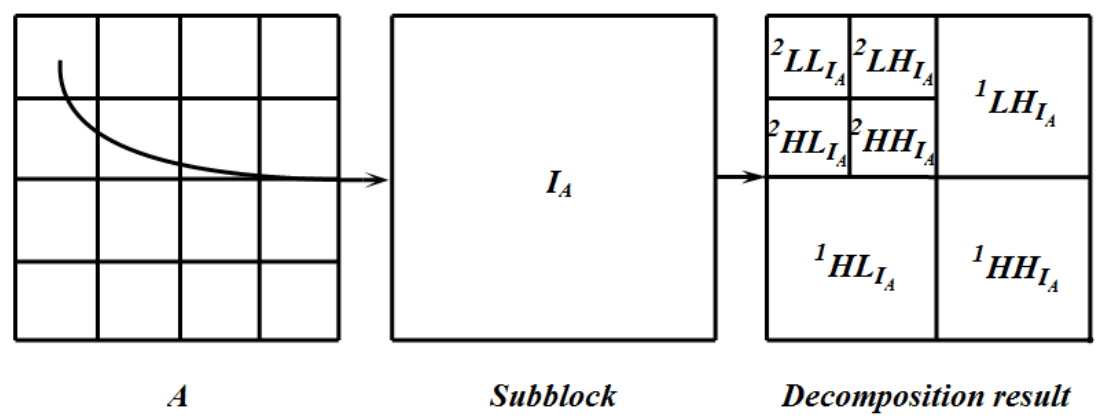

Figure 2 Decomposition schematic diagram of the original image $A(n=2)$

The fusion rules are playing a very important role during the fusion process, so the selection of fusion rules is crucial, it directly affects the effect of the fusion image. To obtain satisfying fusion results, this paper also presents an improved fusion rule according to the characteristics of low frequency and high frequency components obtained by the W-transform.

Improvement of fusion rule for low frequency part. After decomposing the input images, the low frequency component ${ }^{n} L L_{I_{A}}$ contains the average image information and is the approximation of the original sub block $I_{A}$, it generally represents the contour of image $I_{A}$. In general, coefficients in low frequency band are fused by mean value, which is a simple way of obtaining an output image with all regions in focus. However, the focus of different image is different, that means the amount of information is concentrated in different positions. The mean value method overlooks the difference between images, which will make some information weakened and reduce the contrast. In this paper, we use weighted average method instead of the simple mean value method by introducing information entropy of each as the weights. Information entropy [15] is an index to evaluate the information quantity contained in an image. The greater the information entropy of the image, the higher its quality will be. If the value of entropy becomes higher after fusing, it indicates that the fusion performances are improved. The calculation formula is as follows

$$
E n_{A}=-\sum_{i=0}^{L-1} p_{i} \log _{2} p_{i}
$$

Where $\mathrm{L}$ is the total of grey levels if image $\mathrm{A}, p_{0}, p_{1}, \mathrm{~L} p_{L-1}$ is the probability distribution of each level.

Based on the improved fusion rule, the low frequency transform coefficients of the sub block of the fused image $I_{f}$, which is fused by the sub block $I_{A}$ of source image A and the sub block $I_{B}$ of source image $\mathrm{B}$, are calculate as

$$
\operatorname{coef}\left({ }^{n} L L_{I_{f}}\right)=\frac{E n_{n, I_{A}} \cdot \operatorname{coef}\left({ }^{n} L L_{I_{A}}\right)+E n_{n, I_{B}} \cdot \operatorname{coef}\left({ }^{n} L L_{I_{B}}\right)}{E n_{n, I_{A}}+E n_{n, I_{B}}}
$$

$\operatorname{coef}\left({ }^{n} L L_{I_{A}}\right)$, coef $\left({ }^{n} L L_{I_{B}}\right)$, coef $\left({ }^{n} L L_{I_{f}}\right)$ are the low frequency transform coefficients of the $n$th decomposition layer of sub block $I_{A}, I_{B}, I_{f}$ respectively. $E n_{n, I_{A}}, E n_{n, I_{B}}$ are information entropy of the $n$th decomposition layer of sub block $I_{A}, I_{B}$ of original image $A, B$, respectively.

Improvement of fusion rule for high frequency part. When constructing each transform coefficient for the fused image. We will have to determine which source image describes this coefficient better.

The absolute values of wavelet coefficients in the high bands indicate that the variation of gray value and correspond to salient features such as edges or lines. To some extent, it reflects the contribution degree of the source images to the fused image. Local region energy will be more 
reasonable to extract features of source images by utilizing neighbors' information and relationship between pixels. Generally speaking, large region energy means important image information. Currently, the fusion rule of wavelet domain is mainly divided into two kinds: pixel-based fusion rule; window-based fusion rule. Note that only considering the single pixel or the local region feature may generate noise [16] in the image fusion process. In this paper, we propose an improved fusion rule for the high frequency part by considering not only the single-pixel but also local energy so that the sub block corresponding larger energy can be obtained.

Originally, the local energy of area with center $(n, m)$ in the high frequency band of the $i$ th decomposition layer of image $\mathrm{A}$, is denoted by $E_{i, A}^{j}(n, m)$ and defined as:

$$
E_{i, A}^{j}(n, m)=\sum_{n^{\prime} \in J, m^{\prime} \in K} w_{i, A}^{j}\left(n^{\prime}, m^{\prime}\right)\left[H_{i, A}^{j}\left(n+n^{\prime}, m+m^{\prime}\right)\right]^{2}
$$

Where $j=1,2,3$ indicate the horizontal, diagonal and vertical high frequency bands, respectively, $H_{i, A}^{j}(j=1,2,3)$ are high frequency coefficients: $H_{i, A}^{1}=\operatorname{coef}\left({ }^{i} L H_{A}\right), H_{i, A}^{2}=\operatorname{coef}\left({ }^{i} H H_{A}\right), H_{i, A}^{3}=\operatorname{coef}\left({ }^{i} H L_{A}\right), \quad w_{i, A}^{j}\left(n^{\prime}, m^{\prime}\right)\left(n^{\prime} \in J, m^{\prime} \in K\right) \quad$ are weighted coefficients corresponding to $H_{i, A}^{j}(j=1,2,3)$, which obey Gauss distribution, and $J \times K$ is the size of the local area (such as $3 \times 3,5 \times 5,7 \times 7$ ).

Assume that $I_{A}$ and $I_{B}$ are the arbitrary sub block of images $A$ and $B, E_{i, I_{A}}^{j}$ and $E_{i, I_{B}}^{j}$ are the local energy of high frequency part. The local matching degree between high frequency part $I_{A}$ and $I_{B}$ of the $i$ th decomposition layer of $I_{A}$ and $I_{B}$ is denoted by $M_{i, I_{A} I_{B}}^{j}$ and calculated as:

$$
M_{i, I_{A} I_{B}}^{j}(n, m)=\frac{2 \sum_{n^{\prime} \in J, m^{\prime} \in K} w^{\prime}\left(n^{\prime}, m^{\prime}\right) H_{i, I_{A}}^{j}\left(n+n^{\prime}, m+m^{\prime}\right) H_{i, I_{B}}^{j}\left(n+n^{\prime}, m+m^{\prime}\right)}{E_{i, I_{A}}^{j}(m, n)+(m, n)}
$$

where $w^{\prime}\left(n^{\prime}, m^{\prime}\right)\left(n^{\prime} \in J, m^{\prime} \in K\right)$ are corresponding weights. Let the matching degree threshold be $T$.

When $M_{i, I_{A} I_{B}}<T$, the fusion rule is improved as follows:

(1) At point $(n, m)$, if both the high frequency coefficient and local energy of sub block $I_{A}$ are higher than that of $I_{B}$, the high frequency coefficient of sub block $I_{A}$ is chosen as the high frequency coefficient at of $I_{f}$, which is the sub block fused by $I_{A}$ and $I_{B}$. It is also true for exchanging the position of $I_{A}$ and $I_{B}$.

(2) At point $(n, m)$, if the local energy of $I_{A}$ is greater than that of $I_{B}$, but its high frequency coefficient is less than that of $I_{B}$, the fusion result will not be satisfying if we directly use the high frequency coefficient of $I_{A}$ as the high frequency coefficient of $I_{f}$. Considering that the local energy of $I_{A}$ is greater than that of $I_{B}$, we apply the classic fusion rule to sub block $I_{A}$, which uses the weighted average method to rectify the high frequency at $(n, m)$, where the weights obey the Gauss distribution.

$$
H_{i, I_{f}}^{j}(n, m)=\left\{\begin{array}{l}
H_{i, I_{A}}^{j}(n, m), \quad a b s\left(H_{i, I_{A}}^{j}(n, m)\right) \geq a b s\left(H_{i, I_{B}}^{j}(n, m)\right) \& E_{i, I_{A}}^{j}(n, m) \geq E_{i, I_{B}}^{j}(n, m) \\
\bar{H}_{i, I_{A}}^{j}(n, m), \quad a b s\left(H_{i, I_{A}}^{j}(n, m)\right)<a b s\left(H_{i, I_{B}}^{j}(n, m)\right) \& E_{i, I_{A}}^{j}(n, m) \geq E_{i, I_{B}}^{j}(n, m) \\
H_{i, I_{B}}^{j}(n, m), \quad \operatorname{abs}\left(H_{i, I_{A}}^{j}(n, m)\right)<a b s\left(H_{i, I_{B}}^{j}(n, m)\right) \& E_{i, I_{A}}^{j}(n, m)<E_{i, I_{B}}^{j}(n, m) \\
\bar{H}_{i, I_{B}}^{j}(n, m), \quad a b s\left(H_{i, I_{A}}^{j}(n, m)\right) \geq a b s\left(H_{i, I_{B}}^{j}(n, m)\right) \& E_{i, I_{A}}^{j}(n, m)<E_{i, I_{B}}^{j}(n, m)
\end{array}\right.
$$




$$
\left\{\begin{array}{l}
\bar{H}_{i, I_{A}}^{j}(n, m)=\sum_{n^{\prime} \in J, m^{\prime} \in K} w^{\prime}\left(n^{\prime}, m^{\prime}\right) H_{i, I_{A}}^{j}\left(n+n^{\prime}, m+m^{\prime}\right) \\
\bar{H}_{i, I_{B}}^{j}(n, m)=\sum_{n^{\prime} \in J, m^{\prime} \in K} w^{\prime}\left(n^{\prime}, m^{\prime}\right) H_{i, I_{B}}^{j}\left(n+n^{\prime}, m+m^{\prime}\right)
\end{array}\right.
$$

$H_{i, I_{f}}^{j}(n, m), H_{i, I_{A}}^{j}(n, m), H_{i, I_{B}}^{j}(n, m)$ are the high frequency coefficients at point $(n, m)$ of the $i$ th decomposition layer of sub block $I_{f}, I_{A}, I_{B} \cdot \bar{H}_{i, I_{A}}^{j}(n, m), \bar{H}_{i, I_{B}}^{j}(n, m)$ are weighted average value of high frequency coefficient at point $(n, m)$ of the $i$ th decomposition layer of sub block $I_{A}, I_{B}$ in window $J \times K \quad w^{\prime}\left(n^{\prime}, m^{\prime}\right)\left(n^{\prime} \in J, m^{\prime} \in K\right)$ are corresponding weights which obey the Gauss distribution.

If $M_{A B}(n, m) \geq T$, we use the original method:

$$
\begin{aligned}
& H_{i, I_{f}}^{j}(n, m)=\left\{\begin{array}{l}
W_{\max }(n, m) H_{i, I_{A}}^{j}(n, m)+W_{\min }(n, m) H_{i, I_{B}}^{j}(n, m), E_{i, I_{A}}^{j}(n, m) \geq E_{i, I_{B}}^{j}(n, m) \\
W_{\min }(n, m) H_{A}(n, m)+W_{\max }(n, m) H_{i, I_{B}}^{j}(n, m), E_{i, I_{A}}^{j}(n, m)<E_{i, I_{B}}^{j}(n, m)
\end{array}\right. \\
& \left\{\begin{array}{l}
W_{\min }(n, m)=\frac{1}{2}-\frac{1}{2}\left(\frac{1-M_{i, I_{A} I_{B}}^{j}(n, m)}{1-T}\right) \\
W_{\max }(n, m)=1-W_{\min }(n, m)
\end{array}\right.
\end{aligned}
$$

The reconstruction of the fused image. Image reconstruction is the inverse process of image decomposition. The process of fused image reconstruction is performing inverse W-transform on the fused decomposed levels from top to bottom, i.e. using the low and high frequency of the $i$ th layer ${ }^{i} L L_{I_{f}},{ }^{i} L H_{I_{f}},{ }^{i} H H_{I_{f}},{ }^{i} H L_{I_{f}}$ to reconstruct the low frequency component of the $(i-1)$ th layer ${ }^{i-1} L L_{I_{f}}(i=n, n-1, \mathrm{~L}, 2)$, and use the high and low frequency components of the first layer to reconstruct the sub block $I_{f}$ of the fused image $f$. The fused image $f$ can be obtained by splicing all sub blocks in sequence. The downward reconstruction process is illustrated as:

$$
\begin{aligned}
& \left\{{ }^{i} L L_{I_{f}},{ }^{i} L H_{I_{f}},{ }^{i} H H_{I_{f}},{ }^{i} H L_{I_{f}}\right\} \rightarrow{ }^{i-1} L L_{I_{f}} \\
& \left\{{ }^{1} L L_{I_{f}},{ }^{1} L H_{I_{f}},{ }^{1} H H_{I_{f}},{ }^{1} H L_{I_{f}}\right\} \rightarrow I_{I_{f}} \\
& i=2, \mathrm{~L}, n
\end{aligned}
$$

\section{Experimental results and analysis}

Experimental Setup. To evaluate the effectiveness of the proposed algorithm and fusion rule, we select two groups of multi-focused images: Disk and Pepsi with size $512 \times 512$ to perform image fusion. In the experiments, the proposed algorithm and fusion rule are compared with the classical algorithms of Haar transform and DCT transform, all the Image are divided into sub block with size $32 \times 32$, and each sub block is decomposed into two levels. The degree of the W-transforms employed is one. The experiment is conducted in Matlab R2014a on a PC with Intel(R) Core(TM) i5-3230M CPU/2.60GHz/4G/8.1 bit 64 operating system. 


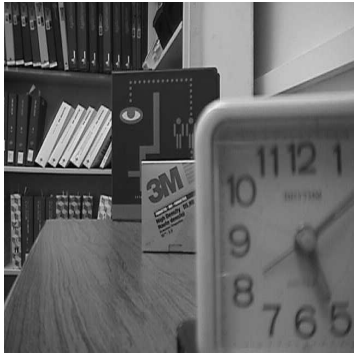

(a) Disk Left focused-image

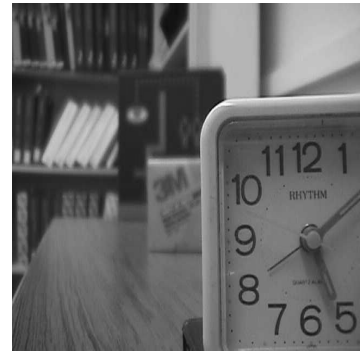

(b) Disk Right focused-image

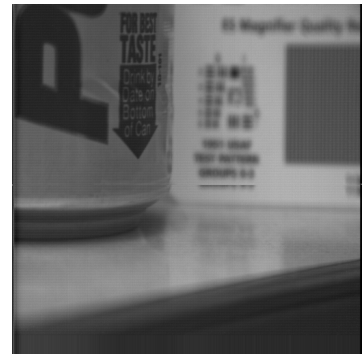

(c) Pepsi Left focused-image

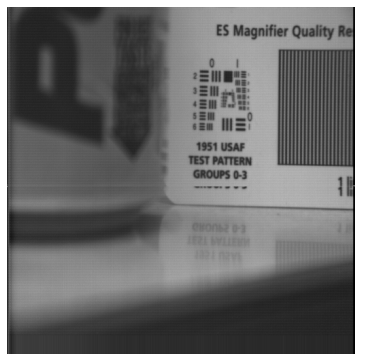

(d) Pepsi Right focused-image

Figure 3 Two groups of Multi Focused Image

Performance Evaluation. There are two main methods to evaluate the fusion performance of different fusion approaches: subjective evaluation and objective evaluation. To quantitatively evaluate the fusion results, we employ four objective index, the Standard Deviatio (SD) [17], Entropy of Information (En), Average Gradient (AG) [15] and Spatial Frequency (SF) [18], to evaluate our methods.

$\mathrm{SD}$ is used for measuring the dispersion degree of the image, and the larger the dispersion degree is, the clearer the image would be. AG is used to describe the changing feature of image texture and the detailed information, larger values of the AG index correspond to higher spatial resolution. En is used to judge the richness of the image information, the higher the entropy is, the more information the image has. And the SF is used to describe the change rate of gray level of the figure. The greater the four indices are, the higher its quality will be. Definition of En is given above in Equ. (6), other three indices are calculate by the following formulas:

$$
\begin{aligned}
& S D=\sqrt{\frac{1}{M \times N} \sum_{m=1}^{M} \sum_{n=1}^{N}(F(m, n)-\bar{F}(m, n))^{2}} \\
& A G=\frac{1}{M N} \sum_{m=1}^{M} \sum_{n=1}^{N} \sqrt{\frac{\Delta F_{x}{ }^{2}(m, n)+\Delta F_{y}{ }^{2}(m, n)}{2}} \\
& S F=\sqrt{R F^{2}+C F^{2}} \\
& R F=\sqrt{\frac{1}{M \times N} \sum_{m=1}^{M} \sum_{n=1}^{N}(F(m, n)-F(m, n-1))^{2}} \\
& C F=\sqrt{\frac{1}{M \times N} \sum_{m=1}^{M} \sum_{n=1}^{N}(F(m, n)-F(m-1, n))^{2}}
\end{aligned}
$$

where $\Delta F_{x}$ and $\Delta F_{y}$ represents the difference of variable $x$ and $y$, respectively, and $R F, C F$ and $S F$ represents the row frequency, column frequency and space frequency of an image, respectively.

Analysis of experimental results. The fusion results of Disk are shown in Fig4, and the fusion results of Pepsi are shown in Fig.5. Among two figures, images labeled as (a), (b), (c) are the fused images obtained by adopting the fusion rule based on the original local energy, and using method of DCT transform (DCT), Haar transform and W-transform of degree one (W1), respectively. Images labeled as (d), (e), (f) are the fused images obtained by adopting the fusion rule based on the improved local energy proposed in this paper, and using method DCT, Haar and W1, respectively. The values of indices of the fused image are presented in Table 1.

Visually, we observed that the fused image obtained by W-transform algorithm is clearer than any of the fused image obtained by DCT, Haar and W1. By comparing images labeled as (a), (b), (c) 
with images labeled as (d), (e), (f), we found that the quality of latter is improved in their clarity, so we can conclude that the fusion rule based on the improved local energy proposed in this paper is more efficient than the fusion rule based on the original local energy. Table 1 shows that the algorithm based on the W-transform is better than the other two algorithms in the four evaluation index: Standard Deviatio, Entropy, Average Gradient and Spatial Frequency. The experiment results demonstrate that the $\mathrm{W}$-system has the superiority in image processing.
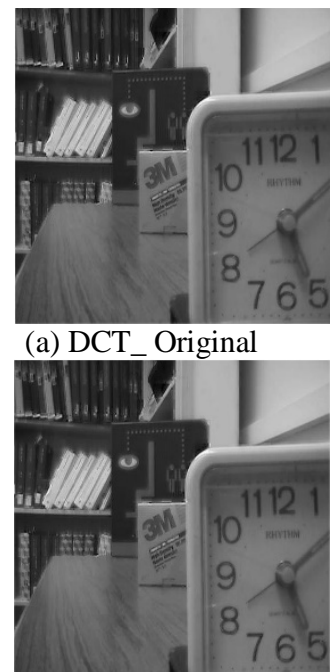

(d) DCT_Improve

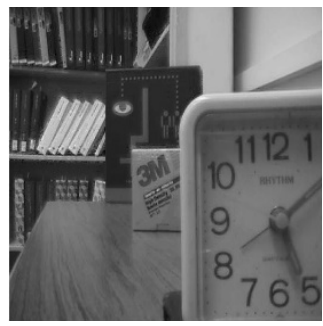

(b) Haar_Original

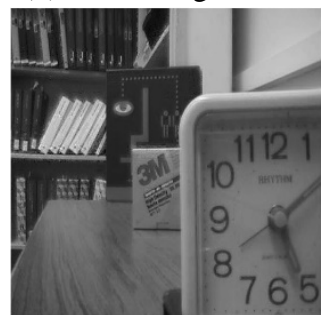

(e) Haar_Improve

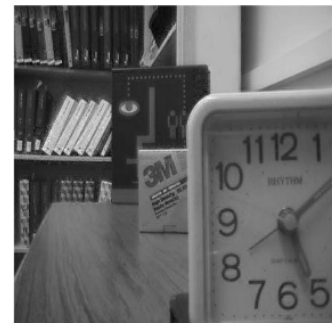

(c) W1_ Original

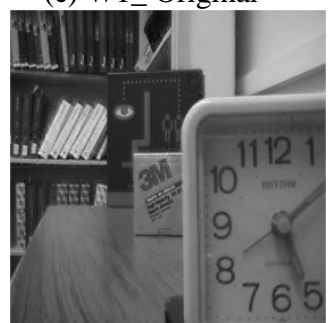

(f) W1_Improve

Figure 4 Disk Sketch map of the image fusion results

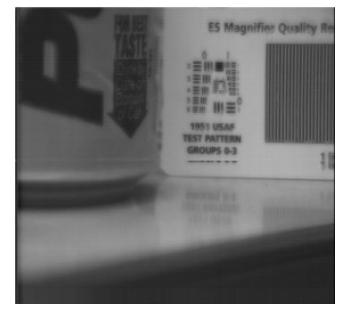

(a) DCT_Original

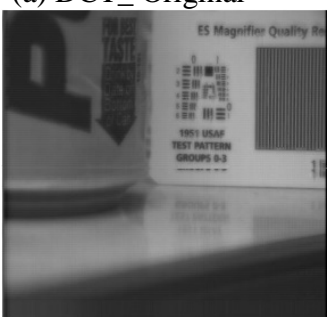

(d) DCT_Improve

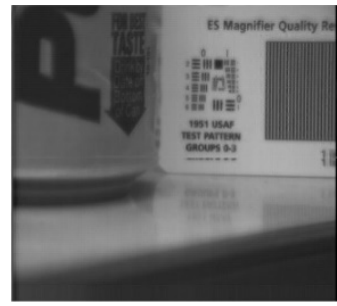

(b) Haar_ Original

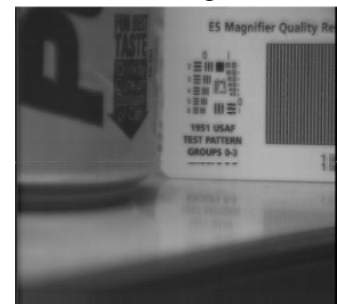

(e) Haar_Improve

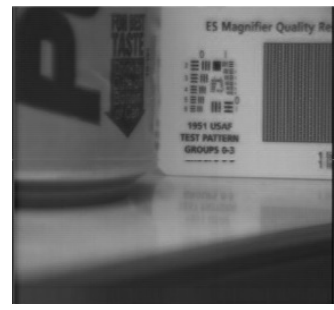

(c) W1_Original

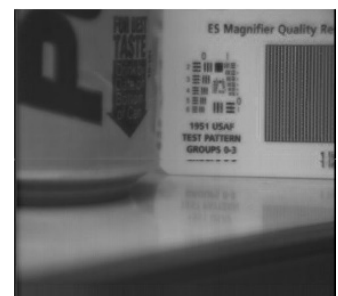

(f) W1_Improve

Figure 5 Pepsi Sketch map of the image fusion results 
Table 1 Comparisons of 4 objective evaluation index in different algorithms

\begin{tabular}{cccccc}
\hline Image & Fusion Method & SD & ENTR & AVEGRAD & SF \\
\hline \multirow{5}{*}{ Disk } & DCT_Original & 43.9723 & 7.2153 & 4.2366 & 11.1228 \\
& Haar_Original & 44.3548 & 7.2229 & 4.3805 & 11.2921 \\
& W1_Original & 44.6179 & 7.2488 & 5.0265 & 12.3069 \\
& DCT_Improve & 43.9987 & 7.2176 & 4.3151 & 11.1498 \\
& Haar_Improve & 44.3653 & 7.2249 & 4.4521 & 11.4344 \\
& W1_Improve & 44.6404 & 7.2529 & 5.2422 & 12.7137 \\
\hline \multirow{5}{*}{ Pepsi } & DCT_Original & 43.9870 & 7.0972 & 4.2824 & 10.9565 \\
& Haar_Original & 44.0536 & 7.0967 & 4.2226 & 11.0974 \\
& W1_Original & 44.1006 & 7.1005 & 4.4463 & 11.4911 \\
& DCT_Improve & 43.9887 & 7.0975 & 4.2872 & 10.9664 \\
& Haar_Improve & 44.0556 & 7.0964 & 4.2336 & 11.1232 \\
& W1_Improve & 44.1068 & 7.1016 & 4.5132 & 11.5982 \\
\hline
\end{tabular}

\section{Conclusions}

In this paper, we propose a W-transform based image fusion method and an improved fusion rules. Similar to the image fusion algorithms based on classical DCT transform and Haar transform, the proposed algorithm is also based on an orthogonal transform. However, the W-transform has the characteristic of reproducibility and can effectively overcome pseudo-Gibbs phenomenon. The image fusion experiment results show that the proposed algorithm outperforms the classical DCT transform and Haar transform in the index of Standard Deviatio, Entropy, Average Gradient and Spatial Frequency, in which the Average Gradient and Spatial Frequency of the W-system respectively increase by $11.70 \%$ and $7.91 \%$ averagely compared with other two fusion methods. The proposed fusion rule which is based on an improved local energy also can improve the performance of image fusion.

\section{Acknowledgement}

This research is supported by National Natural Science Foundation of China under Grant No. 61571046, No. 61372190, National College Student Innovation Training Program (Grant No. 201510022071)

\section{Reference}

[1] YANG Fang-lin, GUO Hong-yang, YANG Feng-bao. Study of Evaluation Methods on Effect of Pixel-level Image Fusion [J]. Journal of Test and Measurement Technology, 2004, 16(4):276-279.

[2] ZHOU Peng, XI Ri-hui, SONG Ling-ling, etc. Realization of Medical Image Fusion Based on Wavelet Transform [J]. Journal of Image and Graphics, 2006, 11(11):1720-1723.

[3] WANG Zeng-min, YANG Zhao-xuan, SU Yu-ting, etc. Image Fusion and Compression Technology in Intelligent Transportation System [J]. Computer Engineering, 2008, 14(14):17-19.

[4] LI Yuan-xiang, YIN Wen, YU Wen-xian, etc. Remote Sensing Image Fusion Based on Sparse Representation: CN, CN103208102 A [P]. 2013.

[5] LI Qin, YU Xin. Study of Image Fusion Methods Appropriated to Biological Images [J]. Acta Optica Sinica,2000, 4(4):494-500. 
[6] MIAO Qi-guang, WANG Bao-shu. Multi-Sensor Image Fusion Based on Improved Laplacian Pyramid Transform [J]. Acta Optica Sinica,, 2007, 9(9):1605-1610.

[7] QIANG Zan-xia, PENF Jia-xiong, WANG Hong-qun. Remote sensing image fusion based on local deviation of wavelet transform [J]. Huazhong Univ.of Sci.\&Tech:Nature Science Edition, 2003, 31(6):89-91.

[8] CHU Heng, ZHU Wei-le. Image fusion algorithms using discrete cosine transform [J].Optics and Precision Engineering, 2006, 2(2):266-273.

[9] Li S, Yang B. Multifocus image fusion by combining curvelet and wavelet transform[J]. Pattern Recognition Letters, 2008, 29(9): 1295-1301.

[10]Upla, KP, Joshi, MV, Gajjar, PP. An Edge Preserving Multiresolution Fusion: Use of Contourlet Transform and MRF Prior. [J] Geoscience and Remote Sensing.2015,53(6),3210-3220.

[11]MI Chen, TANG Xiu-fen, WEI Feng-lan. Image Decomposition and Reconstruction Based on Haar Wavelet Transform [J]. Journal of Network New Media, 2002, 4(2):78-81.

[12]WANG Xiao-chun, SONG Rui-xia. Discrete representation and fast algorithm of new class of orthogonal system [J]. Computer Engineering and Applications, 2008, 44(8):40-44.

[13]LIANG Yan-yan, SONG Rui-xia, WANG Xiao-chun, etc. Complete Orthogonal V-System and It' $\mathrm{S}$ Application in Geometrical Information Reconstruction [J]. JOURNAL OF COMPUTER-AIDED DESIGN \&COMPUTER GRAPHICS, 2007, 7(7):871-875.

[14]LIU Gui-xi, ZHAO Shu-guang, YANG Wan-hai. Multi-sensor Image Fusion Scheme Based on Gradient Pyramid Decomposition [J]. Journal of Optoelectronics Laser, 2004, 12(3):293-296.

[15]JIANG Nian-de, WANG Yao-nan. A New Method for Image Fusion Based on Principal Component Analysis and Wavelet Transforms [J]. Journal of Image and Graphics, 2005, 7(7):910-915.

[16]JIANG Ze-Tao, YANG Yang, GUO Chuan. Study on the Improvement of Image Fusion Algorithm Based on Lifting Wavelet Transform [J]. Journal of Image and Signal Processing, 2015, 4(02): 11.

[17]ZHU Shi-zheng,ZHOU Zuo-feng. Image fusion based on energy of cross band and wavelet transform [J]. Computer Engineering and Applications, 2009, 14(14):164-166.

[18]LI Jian-lin, YU Jian-cheng, SUN Sheng-li. Study of Image Fusion Based on Grad Pyramid Algorithm [J]. Science Technology and Engineering, 2007, 22:5818-5822. 